\title{
LOS RECURSOS DIDÁCTICOS EN LA ENSEÑANZA Y APRENDIZAJE DEL INGLÉS: UNA APROXIMACIÓN EXPLORATORIA
}

\author{
Claudio Díaz Larenas ${ }^{[1]}$ \\ Karen Aguilera Chamorro ${ }^{[2]}$ \\ Romina Fuentes Concha ${ }^{[3]}$ \\ Paula Pérez Becerra ${ }^{[4]}$
}

\section{Resumen}

En este estudio se han identificado las percepciones de 34 profesores de inglés respecto al conocimiento y uso de recursos didácticos en la clase, durante el 2012. A esta muestra se le aplicó un cuestionario tipo escala Likert que permitió explorar el uso de los recursos didácticos desde la perspectiva de los sujetos involucrados. Los resultados han permitido señalar que los profesores del sistema educacional público enfatizaron, mediante los recursos, el desarrollo de vocabulario; mientras los de la dependencia educacional semi-pública estimularon la habilidad de expresión oral y la pronunciación.

Palabras clave: recursos, inglés, profesores, habilidades lingüísticas.

\section{Summary}

This study identified the perceptions of 34 English teachers regarding the knowledge and use of learning resources in the classroom, during 2012. To this sample a questionnaire of the type of Likert scale was applied that allowed us to explore the use of the teaching resources from the perspective of the subjects involved. The results have enabled us to point out that teacher's in the public education system emphasized, through resources, vocabulary development, while those of semi-public educational dependency stimulated the ability of oral expression and pronunciation.

Keywords: Resources, English, teachers, language skills.

\footnotetext{
[1] Doctor en Educación y Magíster en Lingüística. Universidad de Concepción. claudiodiaz@udec.cl

[2] Licenciada en Educación. Universidad Católica de la Santísima Concepción. kaguilera@ucsc.cl

[3] Licenciada en Educación. Universidad Católica de la Santísima Concepción. rfuentes@ucsc.cl

[4] Licenciada en Educación. Universidad Católica de la Santísima Concepción. pperez@ucsc.cl
} 


\section{Introducción}

Los profesores tienen la misión de ser creativos en la sala de clases y motivar al estudiante mediante la enseñanza para así obtener aprendizajes significativos. Para lograr esto, los profesores se esfuerzan en la preparación y selección de distintos recursos didácticos, los cuales son parte fundamental para conseguir la innovación en educación. De hecho, para impactar en la faceta del diseño curricular, los recursos didácticos constituyen un importante campo de actuación.

Si bien el gobierno chileno entrega mayor cantidad de recursos a los establecimientos públicos, es en estos lugares donde el rendimiento escolar es más bajo. Los recursos didácticos en dichos establecimientos no están logrando su objetivo, mientras que en los establecimientos semi-públicos, si bien es cierto que los recursos son menos, los resultados que se obtienen son mejores. Estas razones hacen que este estudio sea relevante; la importancia de los recursos didácticos en la sala de clases, la correcta utilización de éstos, la forma como los profesores están utilizándolos, son respuestas que se pretenden dar a través de este estudio. Este artículo se inserta en el contexto del proyecto "Investigación del conocimiento profesional, las creencias implícitas y el desempeño en aula de estudiantes de Pedagogía en inglés como estrategia de generación de indicadores de monitoreo de su proceso formativo" (FONDECYT No 1120247).

\section{Revisión de literatura}

Importantes cambios educacionales están ocurriendo en Chile. Desde el año 1996, el gobierno está introduciendo una reforma educacional en las escuelas primarias y secundarias para mejorar la calidad del aprendizaje de los estudiantes. En este contexto de reforma, el Ministerio de Educación decide implementar enel 2003, un programa denominado: "El inglés Abre Puertas" y que persigue, entre otros objetivos, mejorar los resultados de aprendizaje. Para lograr este propósito, el Ministerio de Educación de Chile, entre otras acciones, invierte económicamente en recursos didácticos para el desarrollo de la lengua extranjera (Mineduc.; 2004).

\section{Los recursos didácticos en la clase de inglés}

El uso de recursos pueden tener un importante impacto en la enseñanza y el aprendizaje de idiomas (Brown, 2007; Harwood, 2010; Mediano, 2010; Mineduc, 2004). En este marco, los recursos didácticos orientan las fases del proceso educativo. Desde un punto de vista cognitivo, estas fases promueven la construcción de un aprendizaje significativo (Iglesias \& Sánchez, 2007; Mazzeo, 2009; Ur ,2012), clasifica los recursos didácticos, en textos base de la asignatura y materiales complementarios. Por otro lado, García (2008) y Hedgcock y Ferris (2009) agrupan los recursos didácticos en materiales publicados y materiales auténticos. En función del uso de recursos en el 
proceso de enseñanza y aprendizaje del idioma inglés, es posible hacer referencia a los siguientes enfoques y métodos:

Enfoque comunicativo: En este enfoque se enfatiza el uso de materiales de carácter auténtico, es decir, recursos escritos o elaborados por hablantes angloparlantes del idioma (Richards y Rodgers, 2007; Tomlinson, 2011).

Método audiolingual: En este método se utilizan recursos didácticos que se asocian a grabaciones de audio. Los mismos son empleados para exponer información a los estudiantes (Montero, Ruiz y Díaz, 2010; Yule, 2010).

Enfoque gramatical: En este contexto, los principales recursos utilizados son diccionarios y libros de gramática (Lems, Miller, y Soro, 2010; Sánchez, 2011).

\section{Materiales y métodos}

\section{Tipo de investigación}

El diseño de este estudio fue de tipo no-experimental y de carácter exploratorio. Se utilizó una escala Likert con siete dimensiones asociadas al uso de los recursos didácticos. Los participantes de este estudio fueron 34 profesores de inglés, de los cuales 17 de ellos pertenecían a la educación pública, y 17 a la educación semi-pública. El rango de edad de los participantes fue entre 24 y 55 años. La muestra fue de tipo no probabilística, puesto que los encuestados (sujetos voluntarios) se seleccionaron a causa de que obedecían a ciertos criterios de investigación y no dependían de la probabilidad. El cuadro No. 1 presenta una breve síntesis del objetivo del estudio, sus principales variables y el análisis de los datos:

Cuadro No. 1: Datos sobre el análisis

\begin{tabular}{|c|l|}
\hline Objetivo del estudio & $\begin{array}{l}\text { Identificar las percepciones de un grupo de profesores de } \\
\text { inglés respecto al conocimiento y uso de recursos didácticos } \\
\text { en la clase de inglés. }\end{array}$ \\
\hline Variables & $\begin{array}{l}\text { Variables de tipo descriptivas: recursos didácticos en depen- } \\
\text { dencias administrativas públicas y semi-públicas. }\end{array}$ \\
\hline Prueba estadística & No utiliza prueba estadística, análisis de frecuencia. \\
\hline
\end{tabular}

\section{Resultados y discusión}

Las dimensiones analizadas dentro de este objetivo del estudio serán: a) Propósito de los recursos didácticos en la clase de inglés, b) Tipos de recursos didácticos utilizados en la clase de inglés c) Utilización de recursos didácticos en relación a las habilidades y sistemas lingüísticos promovidos en la clase de inglés, d) Tipo de actividades lingüísticas 
que desarrolla el profesor a través del texto de estudio como recurso didáctico y e) Recursos didácticos audiovisuales utilizados en la clase de inglés. Cabe mencionar que para efectos de comprensión de las figuras que se presentarán a continuación el color gris de los gráficos corresponde a la dependencia administrativa pública y el color negro a la dependencia administrativa semi-pública.

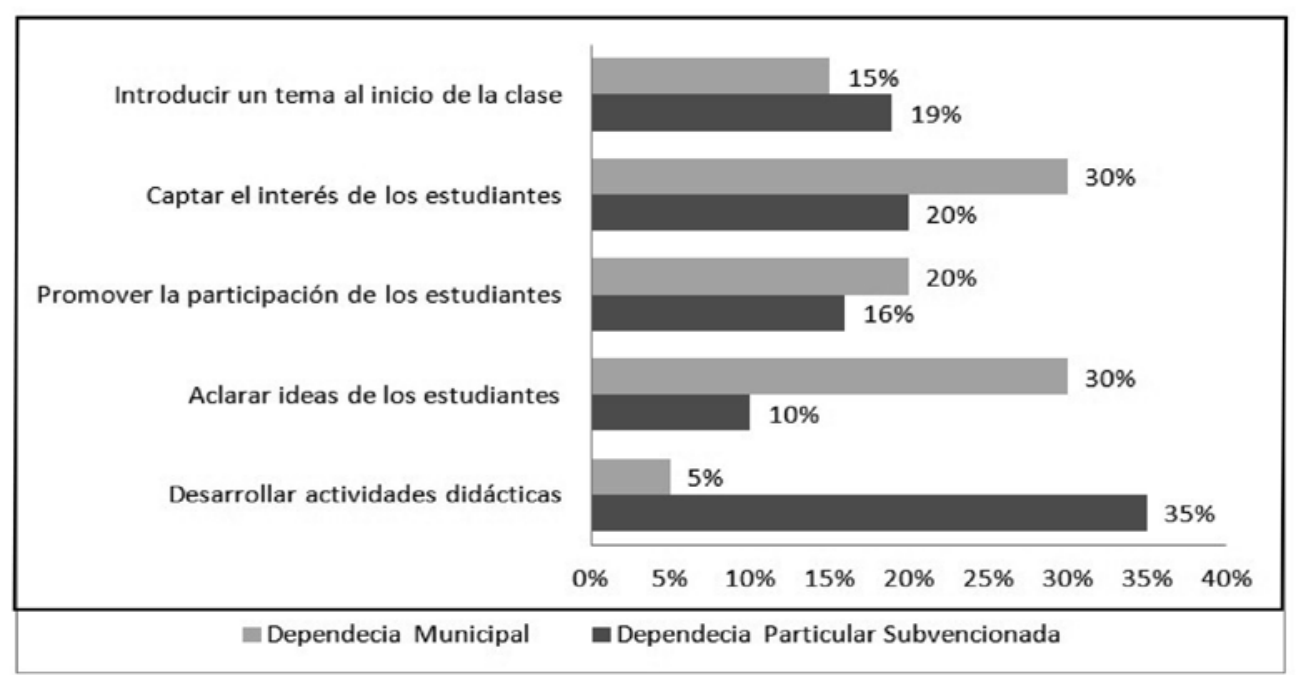

Figura No. 1: Propósito de los recursos didáctico.

En la Figura No. 1. Se observa el porcentaje de conformidad a lo que opinaron los entrevistados respecto al propósito por el cual los profesores utilizan los recursos didácticos en la dependencia pública que es aclarar ideas junto con captar el interés de los estudiantes; esto de debe principalmente a que en este tipo de dependencias el texto de estudio es fundamental en el desarrollo de la clase de inglés; por lo tanto, al usar un nuevo recurso éste sirve para aclarar ideas en el contexto de actividades ya realizadas con el texto. A su vez captan el interés del estudiante, ya que se utiliza un nuevo recurso. En cuanto al propósito de los recursos didácticos en dependencias semi-pública, éste se orienta a desarrollar actividades didácticas. En este tipo de dependencias, las actividades didácticas son más frecuentes. Esto se debe a que los profesores fortalecen las habilidades lingüísticas de sus estudiantes con actividades que motiven su aprendizaje. 


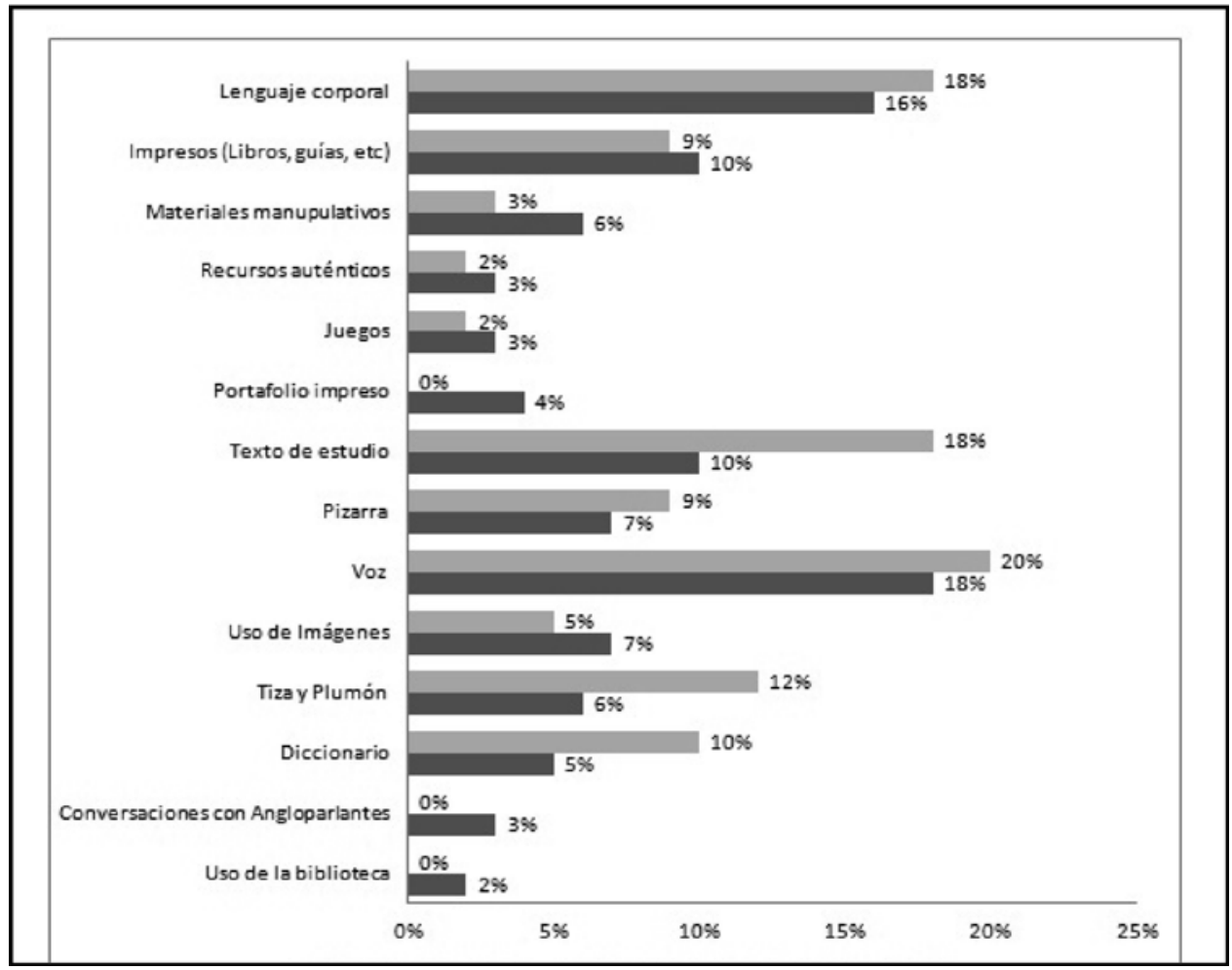

Figura No. 2: Tipos de recursos didácticos utilizados en la clase de inglés.

En la Figura No. 2 se puede observar el porcentaje de los tipos de recursos didácticos utilizados por los profesores de inglés tanto en la dependencia administrativa pública como en la semi-pública. En relación al lenguaje corporal, se observa una estrecha diferencia en porcentaje entre las dos dependencias administrativas. Por un lado, la dependencia administrativa pública presenta un $18 \%$ y, por otro lado, la dependencia administrativa semi-pública un $16 \%$.

Otro tipo de recursos didácticos son los impresos, donde los porcentajes no muestran una gran diferencia en las dos dependencias: un 9\% para la dependencia administrativa pública y un 10\% para la dependencia administrativa semi-pública. En relación con los recursos manipulativos existe una clara diferencia entre dependencias: en la pública son utilizados en un $3 \%$, mientras que en la semi-pública por un $6 \%$. La diferencia en la utilización de recursos auténticos no es tan significativa, ya que en la dependencia administrativa pública es de un $2 \%$ y en la semi-pública es de un $3 \%$. Al igual que los recursos auténticos, la utilización de juegos como recursos didácticos en las clases de inglés no es tan explotada por los profesores. La dependencia administrativa pública sólo presenta un $2 \%$ y la semi-pública un $3 \%$. 
El portafolio impreso es un recurso muy práctico y útil para los estudiantes, pero lamentablemente no está siendo incorporado en estas dependencias, ya que la dependencia administrativa pública señala no utilizar este recurso, y la dependencia administrativa semi-pública lo utiliza sólo un $4 \%$. El texto de estudio como recurso didáctico se utiliza mayoritariamente por la dependencia administrativa pública con un $18 \%$, mientras que en la dependencia administrativa semi-pública es utilizado sólo en un $10 \%$. Un recurso que antiguamente era muy aprovechado por los profesores es la pizarra. Hoy este recurso no es tan utilizado: la dependencia administrativa pública presenta un $9 \%$ y la dependencia administrativa semi-pública presenta un $7 \%$.

El recurso mayormente utilizado por todos los profesores es la voz. La dependencia administrativa pública declara utilizar este recurso en un $20 \%$ y con una pequeña diferencia la dependencia administrativa semi-pública en un $18 \%$. El uso de las imágenes, si bien es un recurso que motiva bastante a los estudiantes, no son muy empleadas por los profesores; la dependencia administrativa pública presenta sólo un 5\%, y la semi-pública un 7\%. La tiza y plumón son recursos que también eran muy utilizados, con el tiempo estos recursos han sido desplazados por nuevos recursos. La dependencia administrativa pública los utiliza en un $12 \%$, y la semi-pública sólo en un $6 \%$. El diccionario también es un recurso que ha sido en cierto punto por la Internet, pero igual existen profesores que declaran utilizarlo en sus clases en estas dependencias: en la pública un 10\% y sólo en un $5 \%$ en la semi-pública.

Lamentablemente, hay recursos como las conversaciones con angloparlantes y el uso de la biblioteca que no son declaradas por la dependencia administrativa pública, apareciendo con un $\% \%$, mientras que en la dependencia administrativa semi-pública, estos recursos son declarados por los profesores sólo en un $3 \%$ y $2 \%$, respectivamente.

Dentro de los tipos de recursos didácticos mayormente utilizados por los profesores de inglés en la dependencia administrativa pública, se destaca la voz, seguida por el lenguaje corporal y el texto de estudio. Estos recursos son esenciales para el aprendizaje de una segunda lengua, pero también se debe considerar que existen otros recursos que complementan este aprendizaje y que estos profesores deben tener presente a la hora de elaborar sus planificaciones de clases. Por otro lado, en la dependencia administrativa semi-pública, al igual que en la pública se destaca la voz, como el recurso más utilizado en la sala de clases, seguida también por el lenguaje corporal.

Claramente, los profesores están utilizando en su mayoría los recursos básicos dentro de la sala de clases de inglés, y postergando los demás recursos que también son de gran importancia en el proceso de enseñanza y aprendizaje. 


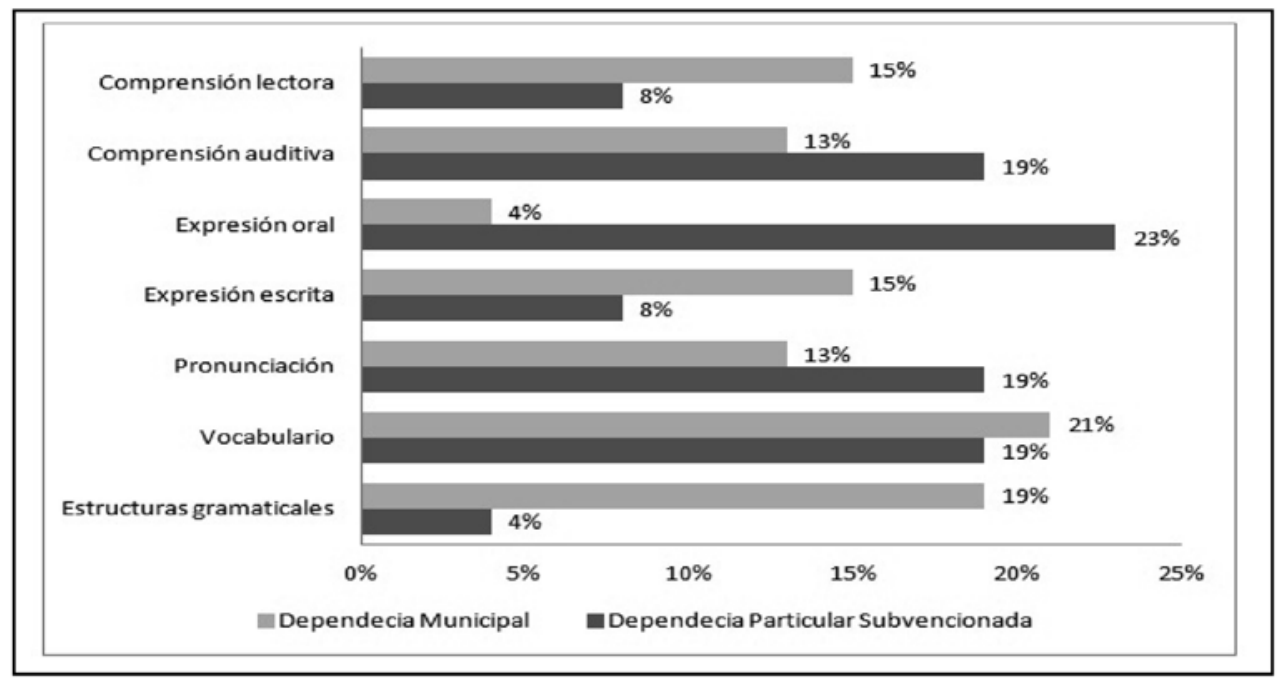

Figura No. 3: Habilidades y sistemas lingüísticos promovidos en la clase de inglés.

En la Figura No. 3, se observa la diferencia en porcentaje, entre las dependencias administrativas pública y semi-pública en relación con las habilidades y sistemas lingüísticos promovidos por los profesores en la clase de inglés. Según se observa en la Figura No. 3, la comprensión lectora es promovida en un $15 \%$ por los profesores públicos y sólo en un $8 \%$ por los semi-públicos. Por otro lado, la comprensión auditiva en la dependencia pública fue de un 13\%, mientras que en la dependencia semi-pública fue de un $19 \%$.

Es importante destacar la gran diferencia que existe entre estas dependencias: sólo un 4\% de los profesores de establecimientos educacionales públicos declara promover la enseñanza de la expresión oral en sus clases, mientras que un $23 \%$ de los profesores de colegios semi-públicos desarrolla esta habilidad. En relación con la expresión escrita un $15 \%$ de los profesores de colegios públicos manifiesta trabajar esta habilidad en sus clases; en cambio, los profesores de establecimientos educacionales semi-públicos sólo la desarrollan en un $8 \%$.

Dentro de los sistemas lingüísticos, las actividades de pronunciación son promovidas en un $13 \%$ por la dependencia pública; por el contrario, en la dependencia semi-pública presenta un 19\%. Por otro lado, las actividades de vocabulario son promovidas, mayoritariamente con un $21 \%$ por la dependencia pública, y en un $19 \%$ por la dependencia semi-pública. Finalmente, en las actividades de estructura gramatical, se observa una gran diferencia entre estas dependencias. Dentro de la dependencia pública, estas actividades forman parte de un 19\%, mientras que en la dependencia semi-pública este tipo de actividades es promovida sólo en un $4 \%$. 
Según la Figura No. 4, se establece que las actividades realizadas en las clases de inglés dentro de las dependencias públicas están orientadas mayoritariamente al aprendizaje de vocabulario y estructuras gramaticales. Esto responde a que las clases se basan principalmente en actividades con el texto de la asignatura, el cual facilita el trabajo de vocabulario y estructuras gramaticales. En cuanto al aprendizaje de vocabulario en las dependencias semi-públicas, la realidad no es distinta. Respecto al aprendizaje de la expresion oral se da una gran diferencia, ya que los profesores de los establecimientos educacionales semi-públicos, enfatizan las habilidades comunicativas para enfrentarse a situaciones comunicativas reales y puedan interactuar sin problemas.

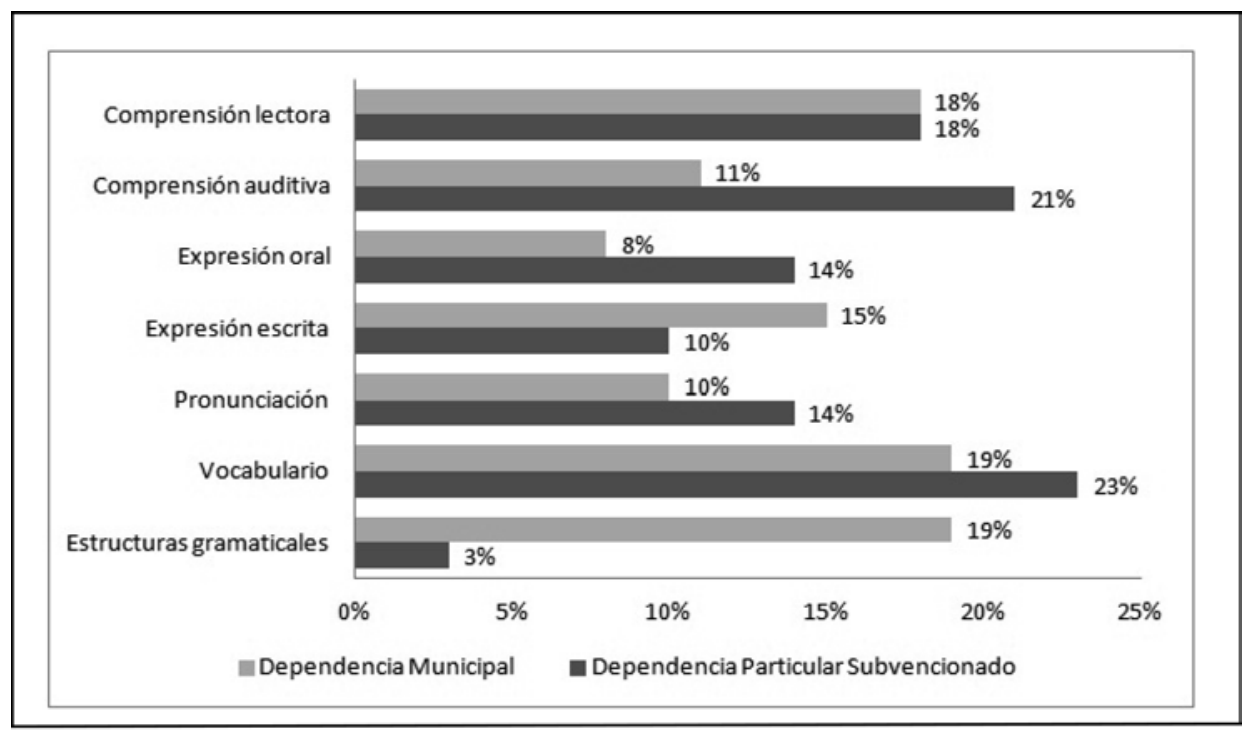

Figura No. 4: Tipo de actividades lingüísticas que desarrolla el profesor a través del texto de estudio.

En la Figura No. 4 se puede observar las distintas actividades lingüísticas que el profesor de inglés realiza en la sala de clases con el uso del texto de estudio. Del total de profesores encuestados, los porcentajes arrojados fueron los siguientes. En relación con la comprensión lectora, tanto la dependencia pública como la semi-pública presentan un $18 \%$. En las actividades de comprensión auditiva, se puede observar que en la dependencia pública un $11 \%$ de los profesores declaran realizar este tipo de actividades, mientras que en la semi-pública un $21 \%$.

Una gran diferencia en porcentaje se presenta en las actividades de expresión oral, ya que sólo en un $8 \%$ estas actividades son promovidas por la dependencia pública, y en un $14 \%$ por la semi-pública. Las actividades de expresión escrita presentes en los textos de estudio de inglés son promovidas en un $15 \%$ por la dependencia pública, y por un porcentaje inferior de $10 \%$ en la semi-pública. 
Dentro de las actividades lingüísticas que presentan los textos de estudio se encuentran las actividades de pronunciación, en un 10\% en la dependencia pública y con un porcentaje de $14 \%$ en la semi-pública. Otra actividad presente en el texto de estudio es la de vocabulario, que tiene un rol importante en el aprendizaje del inglés. Los profesores declaran desarrollar este tipo de actividades en un $19 \%$ en la dependencia pública y en un $20 \%$ en la semi-pública. Por último, se encuentran las actividades de estructuras gramaticales. En la dependencia pública las actividades de estructura gramatical se posicionan con un $19 \%$, lo que refleja una clara diferencia con la dependencia semi-pública donde esta actividad se desarrolla sólo en un $3 \%$.

Si bien el texto de estudio es el recurso más utilizado por los profesores de inglés en los establecimientos educacionales públicos, según lo declarado por los encuestados, éste se usa principalmente para realizar actividades de aprendizaje de vocabulario y estructuras gramaticales. Esto puede deberse a que el texto proveniente del Ministerio de Educación proporciona gran cantidad de conocimientos, pero descuida el trabajo de las habilidades comunicativas de la lengua. Además, las actividades de tipo comunicativas están diseñadas para estudiantes que alcanzan los objetivos secuencialmente año a año y sin dificultades; de lo contrario, deben ser adaptadas por el profesor, lo cual significa trabajo extra. En el caso de las dependencias semi-públicas trabajan con el texto de estudio, principalmente las actividades referidas al aprendizaje de vocabulario, seguidas por las actividades de comprensión auditiva.

Cabe destacar que los establecimientos educacionales semi-públicos usan textos de estudio complementarios al entregado por el Ministerio de Educación.

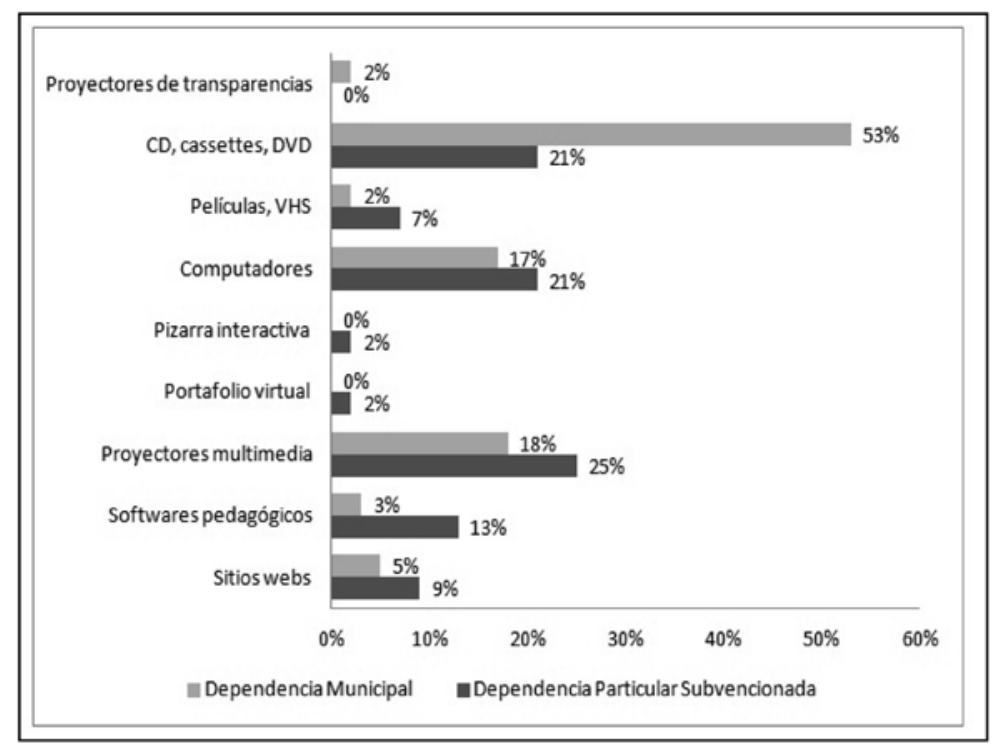

Figura No. 5: Recursos audiovisuales más utilizados en la clase de inglés. 
En la Figura No. 5, se puede observar los distintos porcentajes que arrojó el instrumento aplicado a los profesores de inglés. En primer lugar, se encuentran los proyectores de transparencias, los cuales fueron declarados sólo en un $2 \%$ por la dependencia pública, mientras que en la dependencia semi-pública no son utilizados. Es evidente que este recurso ya no está siendo utilizado por los profesores de inglés, puesto que el rol que éste cumplía es reemplazado por nuevas y más avanzadas tecnologías.

Luego en los materiales sonoros como CD, cassettes, DVD; se observa una gran diferencia, ya que en la dependencia pública, éstos son utilizados en un $63 \%$, mientras que en la dependencia semi-pública sólo en un $21 \%$. Uno de los recursos didácticos poco utilizados por los profesores de inglés son los materiales audiovisuales, como películas en formato VHS, puesto que sólo es utilizado en un $7 \%$ por las dependencias semi-públicas, mientras que en la dependencia pública los profesores encuestados declararon sólo utilizar este recurso en un $2 \%$.

Los computadores, son un recurso importante dentro del proceso didáctico debido a la gran cercanía y el apoyo que brindan a los estudiantes. Sin embargo, aún la totalidad de los profesores no los utilizan. En la dependencia pública son utilizados sólo en un $11 \%$, y en la dependencia semi-pública, en un $19 \%$.

Dentro de los adelantos tecnológicos se encuentra la pizarra interactiva. Aunque este recurso no ha sido masificado aún, posiblemente por su elevado costo económico. La utilización de este recurso fue declarado sólo en un $2 \%$ por la dependencia semipública, y en un o\% por la dependencia pública. Algo similar ocurre con el portafolio virtual, este recurso no es utilizado por los profesores encuentados de la dependencia pública, en consecuencia obtiene un o\%, y sólo es utilizado en un $2 \%$ por la dependencia semi-publica.

Un recurso que está siendo cada vez más utilizado en la sala de clases son los proyectores multimedia. Esto puede ser debido a la gran ayuda que éstos entregan a los profesores a la hora de desarrollar sus clases, en un $18 \%$ son utilizados por los profesores de la dependencia pública y en un $25 \%$ por la dependencia semi-pública.

El uso de computadores, trae consigo el uso de software pedagógicos, los que fueron mencionados sólo en un $3 \%$ por la dependencia pública y en un $13 \%$ por la semi-pública. Por último, se encuentran los sitios webs, altamente utilizados por los estudiantes, pero lamentablemente, no aún en el ámbito educativo. Son utilizados sólo en un $5 \%$ por la dependencia pública y en un $9 \%$ por la semi-pública.

Los recursos audiovisuales son herramientas fundamentales para aprender una lengua extranjera. Uno de los materiales audiovisuales más usados en las clases de inglés dentro de las dependencias públicas son los materiales sonoros, los cuales se componen por $\mathrm{CD}$, radio, casetes entre otros. Esto se explica porque en la dependencia 
pública existen recursos audiovisuales, pero generalmente se encuentran no operativos para el uso cotidiano de los estudiantes. Una razón importante que influye dentro del uso de los recursos didácticos audiovisuales es el conocimiento que los profesores poseen acerca de este tipo de recursos. Muchos de los profesores de dependencias públicas, no saben cómo usar un proyector multimedia, pizarras interactivas y muchas veces ocurre lo mismo con los computadores o distintos software que existen.

Dentro de los recursos audiovisuales que más utilizan los profesores de dependencia pública se encuentran los recursos sonoros, puesto que éstos son más accesibles, fáciles de usar y los $C D$ vienen incluidos en el texto de la asignatura. Por otra parte, los establecimientos educacionales de dependencia semi-pública destinan mayores recursos a la adquisición de tecnologías para el aprendizaje de los alumnos y a su vez esto conlleva al perfeccionamiento de los profesores para usar estas nuevas herramientas.

\section{Consideraciones finales}

Considerando que en el análisis de los datos se ha profundizado respecto de los recursos didácticos utilizados para la enseñanza del inglés, se observa que, en base a los resultados obtenidos a través del cuestionario aplicado a un grupo de docentes de establecimientos educacionales semi-públicos y públicos, se puede decir que existen las siguientes diferencias: Las habilidades lingüísticas mayormente promovidas en la dependencia administrativa pública es el aprendizaje de vocabulario. Mientras que, en las dependencias semi-públicas son la expresión oral y las actividades de pronunciación.

En relación al texto de estudio, la mayoría de los profesores encuestados de las dependencias administrativas públicas, utiliza el texto de estudio de la asignatura de inglés para realizar actividades de vocabulario y de estructuras gramaticales. Por otro lado, en las dependencias semi-públicas, se enfatizan las actividades de comprensión auditiva y aprendizaje de vocabulario.

En cuanto a la utilización de los recursos didácticos audiovisuales en la clase de inglés, se puede señalar que en las dependencias públicas se utilizan mayormente los casetes, CD, DVD. En cambio, en las dependencias semi-públicas los proyectores multimediales muestran un uso importante.

\section{Lista de referencia}

Brown, H. (2007). Teaching by principles. White Plains: Longman.

García, F. (2008). Motivar para el aprendizaje desde la actividad orientadora. Madrid: Ministerio de Educación y Ciencia/Centro de Investigación y Documentación Educativa. 
Harwood, N. (2010). English language teaching materials: Theory and practice. Cambridge: Cambridge University Press.

Hedgcock, J. y Ferris, D. (2009). Teaching readers of English: Students, texts, and contexts. New York/Oxon: Routledge.

Iglesias, M. y Sánchez, M. (2007). Diagnóstico e intervención didáctica del lenguaje escolar. Oleiros: Netbiblo.

Lems, K., Miller, L. y Soro, T. (2010). Teaching reading to English language learners: Insights from Linguistics. New York: The Guilford Press.

Mazzeo, R. (2009). Estudiar: ¿Misión imposible?: Sobre el método de estudio en la escuela y en familia. Madrid: Ediciones Encuentro.

Mediano, F. (2010). Selección y elaboración de materiales educativos. En D. Cervera (Ed.), Didáctica de la tecnología. Barcelona: Ministerio de Educación/Editorial GRAÓ.

Mineduc (2004). La educación chilena en el cambio de siglo: Políticas, resultados y desafíos. Santiago: Ministerio de Educación.

Montero, E., Ruiz, M. y Díaz, B. (2010). Aprendiendo con video juegos: Jugar es pensar dos veces. Madrid: Ministerio de Educación/Narcea.

Richards, J. y Rodgers, T. (2007). Approaches and methods in language teaching. Cambridge: Cambridge University Press.

Sánchez, J. (2011). (Ed.). Nuevas ideas en informática educativa: Memorias del XVI congreso internacional de informática educativa, TISE: Volumen 7. Santiago: Universidad de Chile.

Tomlinson, B. (Ed.). (2011). Materials development in language teaching. Cambridge: Cambridge University Press.

Ur, P. (2012). A course in language teaching. Cambridge: Cambridge University Press.

Yule, G. (2010). The study of language. Cambridge: Cambridge University Press. 